\title{
Une nouvelle forme de capitalisation des connaissances grâce à l'archéologie industrielle avancée
}

A new way for capitalizing knowledge by means of advanced industrial archaeology

Florent Laroche

\section{OpenEdition Journals}

Édition électronique

URL : http://journals.openedition.org/dht/186

DOI : $10.4000 /$ dht. 186

ISSN : $1775-4194$

Éditeur :

Centre d'histoire des techniques et de l'environnement du Cnam (CDHTE-Cnam), Société des élèves du CDHTE-Cnam

\section{Édition imprimée}

Date de publication : 1 décembre 2009

Pagination : 51-60

ISBN : 978-2-9530779-4-0

ISSN : 0417-8726

Référence électronique

Florent Laroche, « Une nouvelle forme de capitalisation des connaissances grâce à l'archéologie industrielle avancée ", Documents pour l'histoire des techniques [En ligne], 18|2 ${ }^{\mathrm{e}}$ semestre 2009, mis en ligne le 08 novembre 2010, consulté le 07 septembre 2020. URL : http://journals.openedition.org/dht/ 186 ; DOI : https://doi.org/10.4000/dht.186 


\title{
Une nouvelle forme de capitalisation des connaissances grâce à l'archéologie industrielle avancée
}

\author{
Florent Laroche \\ École centrale de Nantes - IRCCYN (UMR CNRS 6597)
}

\begin{abstract}
RÉSUMÉ
Dans un contexte actuel d'accélération du progrès et de rentabilisation des innovations, les environnements industriels sont transférés, transformés, abandonnés... C'est la connaissance technique de l'humanité qui disparaît. L'approche développée dans nos recherches consiste à « renverser l'axe des temps de la conception " et à proposer, à partir de l'objet technique et des informations dont on dispose et grâce à l'utilisation d'outils, une capitalisation et une modélisation numérique en vue d'une remise en situation d'usage virtuelle : c'est l'archéologie industrielle avancée. Nous analysons d'abord les enjeux liés à l'utilisation d'un tel outil virtuel. Nous détaillons ensuite la méthodologie développée pour « virtualiser » notre patrimoine technique. Plusieurs exemples et cas d'études menés par notre équipe de recherche viendront illustrer la démarche. Enfin, nous exposons les possibilités et les impacts de cette nouvelle façon de mener l'histoire des sciences et des techniques.
\end{abstract}

Résumés et mots clés en anglais sont regroupés en fin de volume, accompagnés des mots clés français

$P^{2}$ our optimiser sa création de valeur, l'entreprise adapte sans cesse son mode de fonctionnement et ses outils de production. Les machines considérées comme obsolètes carnerépondant plus àla demande sont ainsi arrêtées, remisées voire même démantelées. Les évolutions géopolitiques qui affectent les systèmes techniques classiques de production menacent d'une perte de mémoire les pays ayant eu un passé industriel. Certains sites disparaissent et les savoirfaire qui leur étaient associés aussi. L'absence de protection de ce patrimoine technique et industriel pose aujourd'hui question, d'autant plus que cette culture technique, dévalorisée, peut s'avérer riche de potentiel, par exemple en étant transposée à des domaines d'applications connexes. Sauvegarder, analyser et comprendre les objets de notre patrimoine passé peut permettre de les transformer en capital présent et devenir une réelle source d'innovation à disposition des industriels.

C'estl'enjeu de l'archéologie industrielle avancée, nouvelle discipline, à la croisée des sciences humaines et sociales et des sciences l'Ingénieur, telle que nous l'avons mise en œuvre dans notre doctorat'. C'est aussi le sens de notre enseignement actuel à l'École centrale de Nantes, couplant les cours traditionnels de mécanique et l'histoire des techniques et essayant d'associer les recherches menées sur les objets du temps présent à la transposition des « connaissances du passé en connaissances contemporaines $»$.

Nous nous proposons de présenter les lignes directrices de cette nouvelle archéologie, en évoquant des finalités, puis les modalités que nous

\footnotetext{
1 Florent Laroche, "Contribution à la sauvegarde des objets techniques anciens par l'archéologie industrielle avancée : proposition d'un modèle d'information de référence muséologique et d'une méthode inter-disciplinaire pour la capitalisation des connaissances du patrimoine technique et industriel ", thèse de doctorat sous le direction de Alain Bernard et Michel Cotte, soutenue en décembre 2007 à l'École centrale de Nantes / Université de Nantes, 563 p., récompensée par le prix de la Recherche universitaire 2008 (journal Le Monde). Le manuscrit peut être téléchargé sur http://florent.laroche.free.fr
} 
avons développées dans notre équipe de recherche et explicitées dans notre doctorat. Nous décrirons ensuite des applications concrètes, avant d'analyser les multiples perspectives ouvertes par ce mode de restitution des techniques passées.

\section{Un nouveau domaine : l'archéologie industrielle avancée} En termes de sauvegarde patrimoniale, la priorité est en général accordée aux approches d'architectures industrielles. S'orientant vers les stratégies de gestion des territoires et des paysages, une moindre mesure est accordée à l'héritage des machines, des processus industriels ou des savoir-faire ouvriers.

Cette situation se comprend aisément :

- en raison d'une meilleure conservation fréquente du contenant bâti de l'industrie au détriment du contenu en machines, rapidement revendues ou ferraillées,

- mais également par les nécessités souvent impérieuses de la restructuration urbaine et des projets de réutilisation et de réaffectation entrepris par les collectivités.

À ce jeu, les machines, les usines, les processus de fabrication qui ont forgé notre économie sont oubliés, détruits. Le fait est d'autant plus grave que les sites industriels ont été des lieux de production et d'exploitation de connaissances - un acquis immatériel qui n'a fait que croître depuis des centaines d'années. Demeurant à l'état de vestiges, ces sites recèlent en fait un savoir-faire immatériel fondamental pour notre culture.

Cherchant à faire face à ces pertes de données, certaines entreprises tendent à reconsidérer leur point de vue et se sont lancées dans le vaste chantier du knowledge management. Depuis quelques années, la capitalisation des connaissances est devenue une application à la mode dans les milieux industriels contemporains. Comme l'a dit Tom Stewart en 1997, journaliste pour le magazine Fortune : "L'actif le plus précieux des entreprises est son capital intellectuel $\|^{2}$.

Pour autant, la faible place de la culture technique en France ne favorise pas, pour l'instant, l'approche que nous développons pour restituer la valeur de notre patrimoine technique etindustriel. Certaines démarches de réhabilitation architecturale revendiquent même une coupure avec le passé privilégiant les cotations immobilière et foncière à la valeur industrielle intrinsèque. Les conséquences sont nombreuses et peuvent se situer entre deux pôles opposés :

- pour les branches professionnelles : c'est une disparition irréversible de compétences techniques et industrielles,

2 Tom Stewart, «Intellectual capital: the new wealth of organizations", Fortune, 1997, 150 p.
- selon le point de vue des observateurs : l'oubli de la valeur ajoutée potentielle que pourrait apporter une culture technique et industrielle pleinement assumée.

Une certaine urgence est donc associée à ce risque de déperdition de connaissances. De plus, ce que les générations en activité peuvent encore comprendre en détail n'appartiendra plus aux systèmes techniques des siècles futurs lorsque ce savoir-faire deviendra patrimonial. En effet, la conservation et la vulgarisation de ces objets dans les musées et les sites font transparaitre un vieillissement intrinsèque de l'information technique. Une nouvelle forme de muséologie devient nécessaire afin que ce savoir-faire immatériel soit pérennisé, mais aussi le contexte de travail. Comme le souligne Paul Rasse, " on est bien loin de l'usine et de l'atelier, du bruit et de la poussière, de la fatigue et de la sueur, de la fureur des luttes et de la violence des rapports sociaux... $11^{3}$.

Ne pouvant suivre la ligne des priorités industrielles, il revient alors aux universitaires, en collaboration avec les conservateurs de musées, les historiens et les experts en patrimoine d'apporter des éléments de réponse et de mettre en œuvre des moyens pour conserver et comprendre - immortaliser - ces savoirfaire matériels et immatériels à vocations scientifique, technique et industrielle.

Dans cette logique d'anticipation de la perte des savoir-faire scientifiques et techniques, notre équipe de recherche a formalisé une méthodologie et l'a expérimentée sur plus d'une dizaine de cas d'études. $\mathrm{Ce}$ projet a sous-tendu notre thèse de doctorat menée en cotutelle sciences humaines et sociales / sciences pour l'ingénieur.

L'approche développée consiste à « renverser l'axe des temps de la conception ॥. A partir de l'objet technique et des informations dont on dispose (vestiges archéologiques, photos, textes, plans 2D...), nous reconcevons l'objet technique et en créons une maquette numérique. Nous avons donc mis en place une démarche intégrant l'ensemble des outils numériques utilisés dans l'industrie contemporaine (comme la numérisation 3D, les logiciels de conception assistée par ordinateur, la réalité virtuelle...). Dès lors, il ne s'agit pas d'obtenir une simple modélisation géométrique en trois

3 Paul Rasse, "Communication et muséologie des techniques 1), colloque REMUS (12-13 décembre 1991, Paris, Palais de la découverte), REMUS la muséologie des sciences et des techniques, ouvrage d'actes édité par l'Office de coopération et d'information muséographiques, 1993, pp. 18-23. 


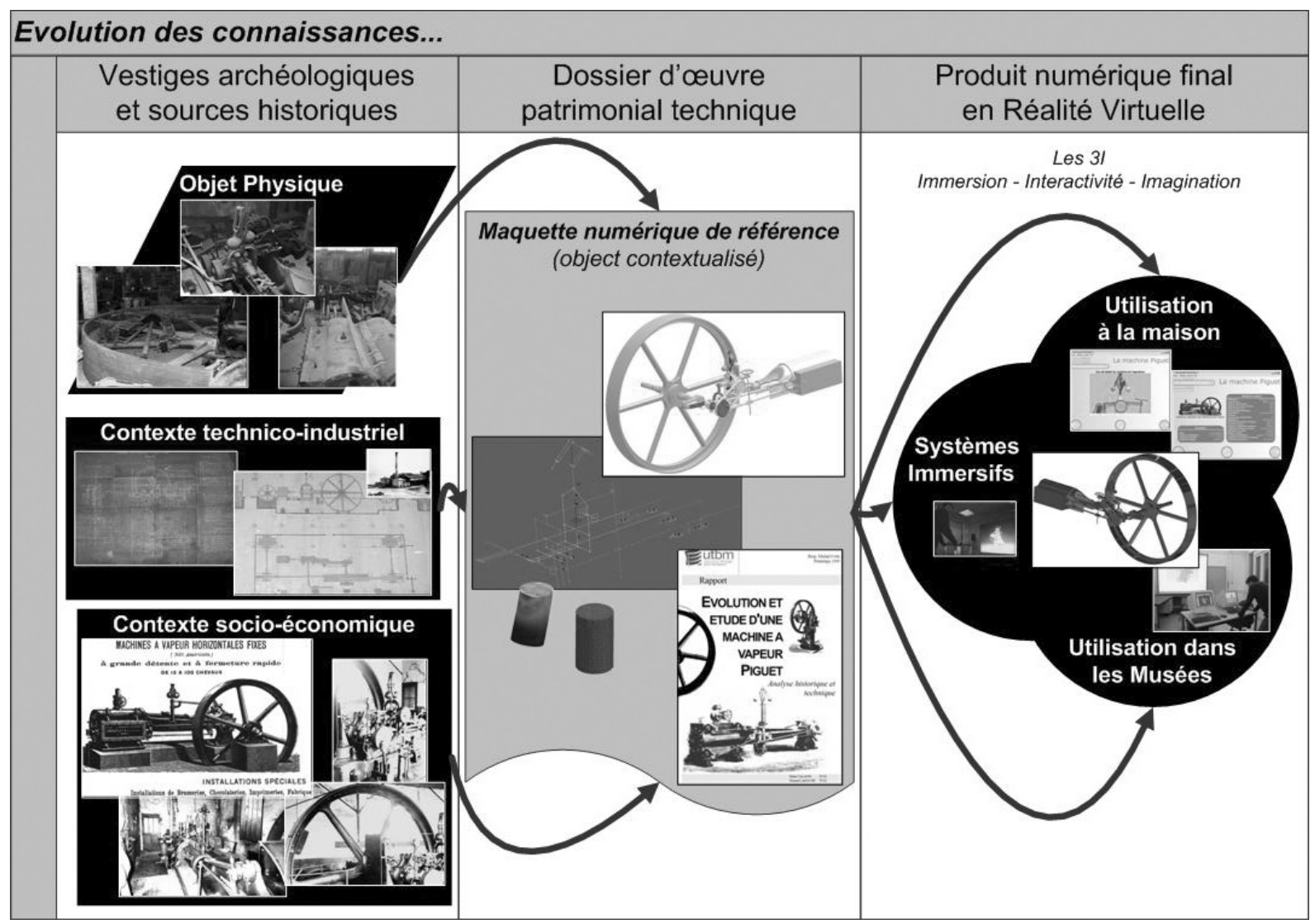

fig. 1 - Les différentes étapes de l'archéologie industrielle avancée.

dimensions de la machine ; c'est tout le contexte de l'objet technique qui est étudié : description des organes fonctionnels, des flux de matière et des fluides, des interactions homme/système... Le travail des sciences humaines est essentiel car le niveau de restitution du contexte (" dézoom ») peut même atteindre l'étude de l'environnement manufacturier dans son ensemble.

Couplant le point de vue technologique de l'ingénieur avec la vision patrimoniale d'un historien des techniques, c'est un nouveau métier et une nouvelle discipline qui apparaissent. Ce n'est donc plus seulement de l'archéologie car les études se focalisent sur des usines et non plus que sur des monuments historiques. Mais c'est bien plus que de l'archéologie industrielle car désormais les machines et la technique sont à l'honneur. II n'est pas pour autant question d'isoler ces objets, il faut retrouver les savoir-faire techniques qu'ils véhiculaient et les compétences développées à travers leur usage - une thématique qui rejoint les méthodes de knowledge management (KM). C'est ce que nous appelons I'AIA, l'archéologie industrielle avancée.

Destinées à des fins de vulgarisation scientifique, muséographique et didactique, ces images virtuelles ne remplacent jamais le réel. Ces modèles numériques ne sont que des illustrations ou des évocations et ne doivent pas être considérés comme un moyen de conservation de l'information au sens rigoureux du terme mais plutôt comme un support à la compréhension. Les éléments apportés par ce travail de recherche n'ont pas pour but de proposer un miracle technologique virtuel en lieu et place des actions de conservation classiques. II s'agit de prendre conscience des limites imposées par les faits et par les pratiques du patrimoine, pour les compléter, les renforcer etles accompagner. Par ailleurs, non seulement le statut numérique possède ses propres limites, mais il pose à son tour de nouveaux problèmes spécifiques, tant pratiques qu'épistémologiques. La figure 1 illustre les différentes étapes de cette démarche.

La méthodologie développée dans l'archéologie industrielle avancée permet la constitution du dossier d'œuvre patrimoniale technique en capitalisant les connaissances du passé sous une forme numérique et en les repositionnant virtuellement en situation d'usage à des fins de muséographie et de valorisation. II s'agit du rétro-processus de conception patrimoniale.

Le schéma suivant démontre que les deux points de vue adoptés, celui de l'histoire et celui des techniques, sont convergents. Même si l'on n'utilise pas le même vocabulaire, les outils et les méthodes ne sont pas pour autant non-homogènes. Du moins, nous avons travaillé à créer un champ de recherche commun utilisant la même sémantique à travers cette nouvelle discipline. 


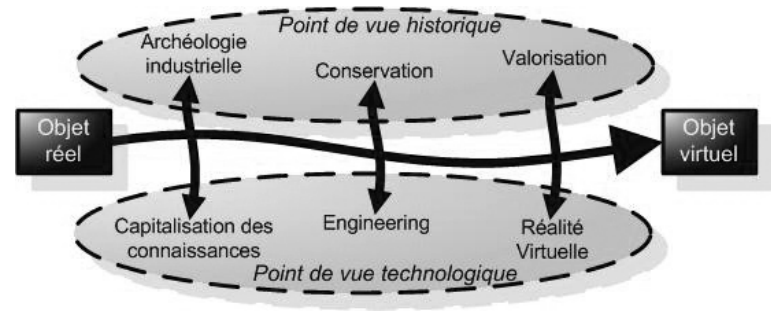

fig. 2 - Méthodologie générale : sciences humaines vs sciences de l'ingénieur.

La méthodologie associée pour « appréhender $\rightarrow$ interpréter $\rightarrow$ restituer $\rightarrow$ valoriser » a été affinée pour obtenir un processus opérationnel et applicable sur le terrain (fig. 2). Afin de transformer un objet réel en un objet virtuel muséographié, nous avons mis en place un rétro-processus de conception patrimoniale. Celui-ci exploite l'intégralité des outils numériques utilisés par les grandes entreprises automobiles, aéronautiques : numérisation 3D, modélisation en conception assistée par ordinateur, simulation de flux, résistance des matériaux... Cependant, pour assurer la complétude de l'étude internaliste, notre méthodologie inclut également une étude externaliste. La combinaison des deux démarches permet alors de décliner et restituer l'objet dans son système technique passé. Pour ce faire, les démarches historiennes et archivistiques doivent s'ouvrir à la vision des technologues; de plus, elles permettre de fournir une information juste et interprétable. En effet, des problèmes de compréhension des mécanismes ou du site industriel peuvent surprendre tout ingénieur confronté à l'utilisation d'une sémantique technique ancienne pour laquelle un dictionnaire historique serait nécessaire. Les archives techniques, les plans et les descriptions de machines sont des documents particulièrement fragiles et disparaissent souvent. Si par chance ces écrits sont retrouvés, ces traces révèlent des informations essentielles et nécessaires à la compréhension de l'objet technique ancien.

Compte-tenu de la complexité des objets techniques étudiés et afin de guider les acteurs du processus de patrimonialisation, il faut pouvoir caractériser de façon exhaustive l'objet technique et son environnement. La complexité des objets induisant une multiplicité des compétences, le projet d'ingénierie patrimoniale requiert la coopération de métiers qui, jusqu'alors, ne collaboraient pas ou peu. De nouvelles formes d'équipes inter-disciplinaires émergent ainsi, disposant d'un référentiel commun afin d'harmoniser les différentes sémantiques. Nous avons donc mis en place un système d'information globalisant pour :

- encapsuler la description de l'objet technique ancien dans son aspect internaliste fonctionnel
- prendre en compte le contexte de l'objet et l'intégrer dans son environnement; ; l s'agit ici du travail fondateur des historiens qui permet de définir l'aspect externaliste des systèmes techniques passés,

- décrire les différentes transformationsintermédiaires de l'objet depuis ses vestiges archéologiques jusqu'aux projets de valorisation multimédia,

- considérer également les aspects humains, les acteurs du passé qui ont utilisé l'objet, ainsi que les acteurs du temps présent impliqués dans le processus de patrimonialisation.

Se situant à l'interface des sciences de l'ingénieur et dessciences humaines etsociales, ce modèle, exprimé dans le langage UML, unified modeling language, est avant tout conceptuel. Le DHRM, digital heritage reference model ou dossier d'œuvre patrimoniale numérique de référence, définit l'objet technique à caractère patrimonial dans ses fonctionnalités et dans son histoire, et à différentes échelles (du boulon à l'usine et depuis l'opérateur passé jusqu'au visiteur de musée). D'un point de vue opérationnel, le DHRM se décline en une maquette numérique de référence, associée à des connaissances externes et au savoir-faire anthropologique. II s'agit d'un nouvel outil de travail muséologique pour lequel la couche technique n'existait pas encore.

Sauvegarder et conserver un objet physique coûte cher pour un musée ; de plus, le démantèlement est parfois impossible (la machine tombant en ruine). Nous proposons de le sauvegarder sous la forme d'un objet virtuel, d'une maquette numérique.

La figure 3 présente le processus global que nous avons mis en place pour capitaliser et valoriser le patrimoine scientifique, technique et industriel.

Passer directement de l'état A à l'état C n'est pas recommandé. En effet, les différentes possibilités de finalités énumérées ci-après pour l'état C montrent qu'il est nécessaire de capitaliser un maximum d'éléments au départ. Ainsi, un état intermédiaire structuré contenant l'ensemble des informations, des données et des connaissances est indispensable : c'est ce que l'on appelle, par extension à la définition muséographique du dossier d'œuvre, le dossier d'œuvre patrimonial technique. Notons que l'appellation anglaise, déjà signalée, est plus signifiante et qu'elle est utilisée largement (DHRM pour digital heritage reference model) ${ }^{4}$. La première phase concerne l'acquisition des données. II s'agit d'un travail classique en histoire des techniques, consistant à rassembler la documentation. La

4 Florent Laroche, Alain Bernard, Michel Cotte, Samuel Deniaud, " A new methodology for a new life of old technical machines ", actes de la conférence internationale CIRP Design Seminar, Alberta, Canada, 2006, 12 p., www.cirp.net 


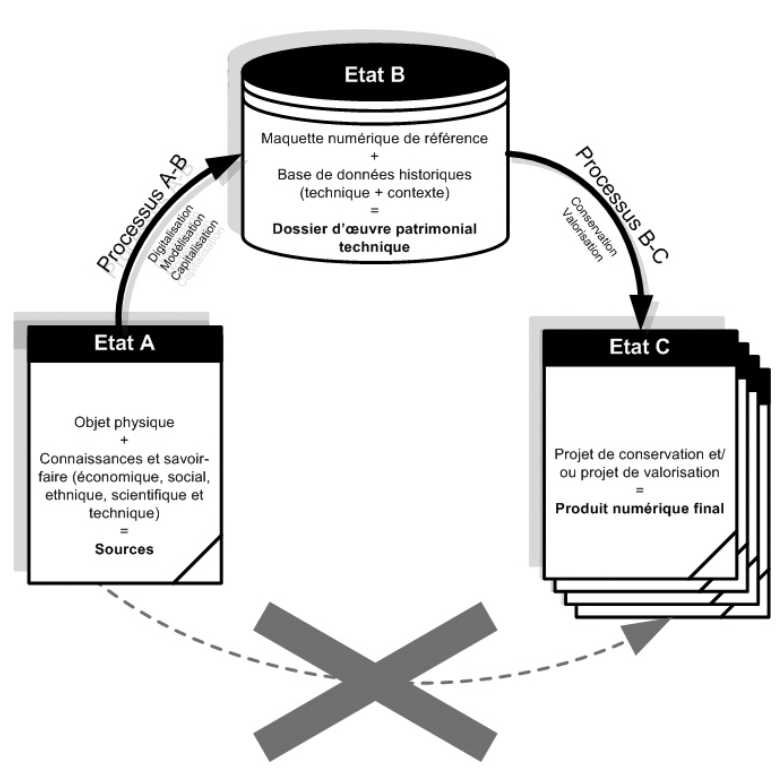

Fig. 3 - Méthodologie générale pour conserver et valoriser le patrimoine technique et industriel.

première catégorie concerne les plans, les données archéologiques, anthropologiques ou ergonomiques, les restes de machines. La deuxième catégorie est constituée par des données économiques, sociales, architecturales, paysagères.

Par la suite, un travail de numérisation des données, de prise de cotes, est mené. Les premiers outils de base sont bien entendu le pied à coulisse et le mètre à ruban. Cependant, afin de gagner en temps de mesure et afin de pouvoir prendre en charge des machines complexes, la numérisation 3D peut être utilisée : théodolite à balayage laser, photogrammétrie, scanner laser 3D avec reconstruction topographique en temps réel. Afin de choisir la meilleure technique en fonction des contraintes de l'environnement et de l'objet patrimonial, nous avons développé un algorithme de décision pour guider la démarche de sauvegarde 5 .

La phase explicitée précédemment est guidée par un méta-modèle conceptuel de données. Ce modèle est également utilisé pour analyser les données numérisées dans le but d'élaborer la maquette numérique de référence de l'état $B$ et ainsi conduire au DHRM. Pour cette phase, nous avons également développé le rétro-processus de patrimonialisation, déjà signaléb.

5 Florent Laroche, Alain Bernard, Michel Cotte, « 3D digitalization for patrimonial machines »), Future of product development, 2007, pp. 397-408, www.cirp.net

6 Florent Laroche, Alain Bernard, Michel Cotte, « Une vision produit-process et sa méthodologie dédiée à la sauvegarde
Le DHRM fait intervenir toutes les données à caractères scientifique, technique et dynamique de la machine. De plus, une base de données à caractère historique lui est associée. Cet ensemble constitue donc le dossier d'œuvre patrimonial technique, permettant la gestion des connaissances.

A partir de l'état de référence constitué précédemment, plusieurs possibilités d'utilisation de cette maquette à des fins de valorisation ont envisageables (état C) : thésaurus virtuel (archives numériques), sauvegarde en archéologie industrielle, utilisation didactique pour experts ou universitaires, reconstruction, valorisation muséographique pour tout type de public. Pour le dernier cas d'application, plusieurs approches peuvent être développées. Au vu de la croissance exponentielle des technologies de réalité virtuelle, nous menons actuellement des recherches d'un point de vue utilisateur afin de cerner au mieux les besoins du public quant à l'appréhension de ce type de technique ${ }^{7}$.

Des projets : le canot d'Indret et les marais salants de Batz-sur-Mer ${ }^{8}$

Appelant de nos vœux une nouvelle génération d'ingénieur-historien ou d'ingénieur-généticien au service de la genèse des produits et de leurs techniques, nous avons expérimenté la méthode sur de nombreux cas d'études. Un axe fort de développement de notre théorie s'appuie sur la formation. Ainsi, l'un des projets pilotes a été mené par une équipe étudiante pluridisciplinaire composée de futurs ingénieurs mécaniciens (École centrale de Nantes), de futurs ingénieurs thermiciens

du patrimoine technique et industriel sous une forme virtuelle $॥$, MICADO Ingénierie numérique collaborative, 2009, à paraître. 7 Florent Laroche, Alain Bernard, Michel Cotte, " A new approach for preserving the technical héritage ",, colloque scientifique international VRIC, Salon Laval virtual, Laval, France, 11 p., 2006, disponible en consultation à la BNF sous le numéro ISBN 2-287-48363-2.

8 Ces projets sont le fruit d'une coopération entre le laboratoire IRCCYN de I'Ecole centrale de Nantes (UMR CNRS 6597) et le Centre François Viète d'Histoire des sciences et des techniques de l'université de Nantes (EA 1161). La reconstitution du canot à vapeur de 1860 est le travail d'une équipe. Pour les aspects pédagogiques, ont également participé à ce projet : I'IUT de Nantes et I'Ecole polytechnique de l'université de Nantes. La capitalisation et la reconstruction de la laverie de sel de Batz-sur-Mer s'effectue grâce à une coopération étroite avec les conservateurs du Musée sous tutelle de la Communauté d'agglomération de Cap atlantique. Ces projets sont réalisés avec le soutien de l'AIP Primeca Pays de la Loire. 
(École polytechnique de l'université de Nantes), d'étudiants en licence professionnelle (IUT de Nantes) et de professionnels du patrimoine et de l'histoire des techniques. S'agissant d'un projet de recherche mais également à vocation pédagogique, nous avons travaillé en partenariat industriel avec la société DCNS?, spécialisée dans les systèmes navals de défense à haute valeur ajoutée. Ce projet a plus particulièrement été suivi par le site d'Indret (44) du groupe DCNS, site dédié à la propulsion nucléaire.

En septembre 2001, diverses archives de l'entreprise sont rassemblées ; en effet, DCNS dépendait du ministère des Armées ; ainsi, l'intégralité des documents de travail a été conservée selon une logique militaire. Afin de valoriser ce patrimoine, le Pôle historique d'Indret (PHI) a été créé en janvier 2002. Le $\mathrm{PHI}^{10}$ est considéré comme une entité à part entière et est placé sous la coordination du secrétaire général de l'établissement ainsi que du Service communication. Sa mission : sauvegarder le patrimoine historique, industriel et bâti du site créé en 1777 sous tutelle de la Marine. L'ensemble de ce fonds est placé sous le contrôle du conservateur des Archives de la Marine de Lorient.

Parmi les milliers de mètres linéaires d'archives de toutes typologies de sources que le PHI possède, un ancien plan couleurs en grand format à échelle 1:10 a été retrouvé ; il n'est pas daté (fig. 4). Cependant, il s'agirait d'un projetd'élaboration d'un canotà vapeur destiné à la marine française comme en témoigne la dépêche ministérielle du 9 décembre 1860"1. Le 28 août 1862, le rapport de commission chargée des essais du canot est dressé ${ }^{12}$ : ce bateau aurait donc effectivement été construit en 1861-1862 et testé sur la Loire, à titre de prototype.

Afin de valoriser son patrimoine historique industriel, le PHI a décidé d'étudier cet objet en alliant l'histoire technique du canot aux métiers actuels concernés par la propulsion. Le plan original a été restauré et l'étude a commencé ${ }^{3}$. L'objectif

9 www.dcnsgroup.com

10 Dans la suite de cet article, nous utiliserons le terme PHI pour désigner le Pôle historique d'Indret, il s'agit de l'appellation exacte utilisée par l'entreprise DCNS pour nommer ce service interne.

11 Ministère de la Marine, Dépêche ministérielle : la direction du matériel du Ministère de la marine Française à M. le Directeur de l'Établissement de la Marine, Archives du PHI-DNCS, Indret, Dpt 44, 9 décembre 1860.

12 Établissement de la Marine (1862), Correspondance : l'Établissement de la Marine à la direction du matériel du Ministère de la marine, Archives du PHI-DNCS, Indret, Dpt 44, 28 août 1862. 13 Florent Laroche, Michel Cotte, Jean-Louis Kerouanton, Alain Bernard, " L'image virtuelle comme source de connais-

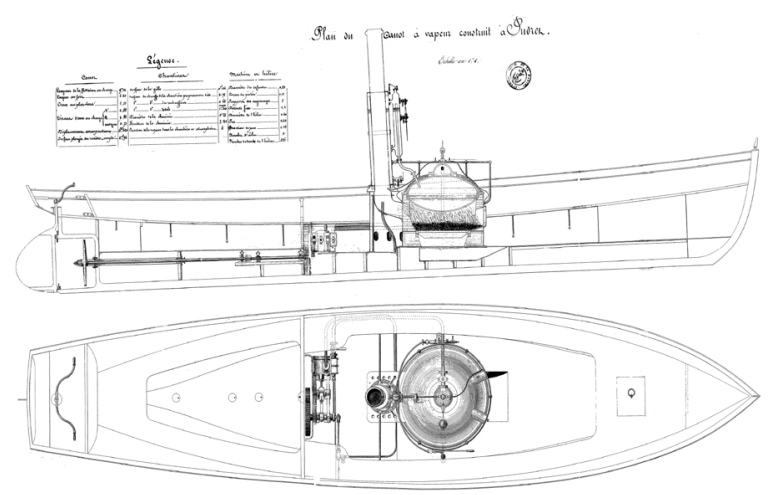

fig. 4 - Canot à vapeur, vue de tribord coupé du plan de 1860 (Établissement de la Marine, Plan du canot à vapeur construit à Indret, échelle 1/10, dessinateur inconnu, Archives du PHI-DNCS, Indret, Dpt 44, n.d., 1860)

fixé était de pouvoir expliquer le fonctionnement du canot, mais également de pouvoir comprendre son dimensionnement, ainsi que les choix techniques réalisés à l'époque et sa place dans l'évolution des bâtiments militaires réalisés ensuite sur le site d'Indret.

Le canot, de dix mètres de long, possède une chaudière en son centre. La sortie du foyer est connectée directement à la cheminée. Cette dernière est renforcée par des armatures métalliques. La machine à vapeur est disposée à l'arrière de la chaudière. Desinstruments de contrôle et des systèmes de sécurité sont également installés sur la chaudière. Mais, à la vue des croquis, des interrogations et des particularités peu communes pour une conception du XIXe siècle apparaissent. C'est, entre autre, le cas de l'utilisation d'une chaudière de type bouilleur. En effet, cette technique n'est plus que brièvement évoquée dans les littératures de l'époque. À cette période, les chaudières tubulaires sont préférées car plus performantes et surtout plus sécurisées. De plus, le cartouche du plan fait mention d'une vitesse de rotation de l'hélice de $420 \mathrm{tr} / \mathrm{min}$. Considérant le profil de l'hélice dessiné, cette valeur met en doute la réelle efficacité du bateau.

La première étude a donc permis de comprendre la partie échange thermique et la machine à vapeur. Puis, les étudiants mécaniciens ont eu pour tâche de réaliserla maquette numérique à partir del'unique plan d'ensemble restauré, en utilisant à titre expérimental

sance pour le patrimoine technique et industriel : Comment allier Histoire et Ingénierie? "1, dans Bertrand Lavédrine dir., Genres et usages de la photographie, $132^{\mathrm{e}}$ congrès national des sociétés historiques et scientifiques (Arles, 2007), Paris, CTHS, 2009, pp. 53-64 (édition électronique). 


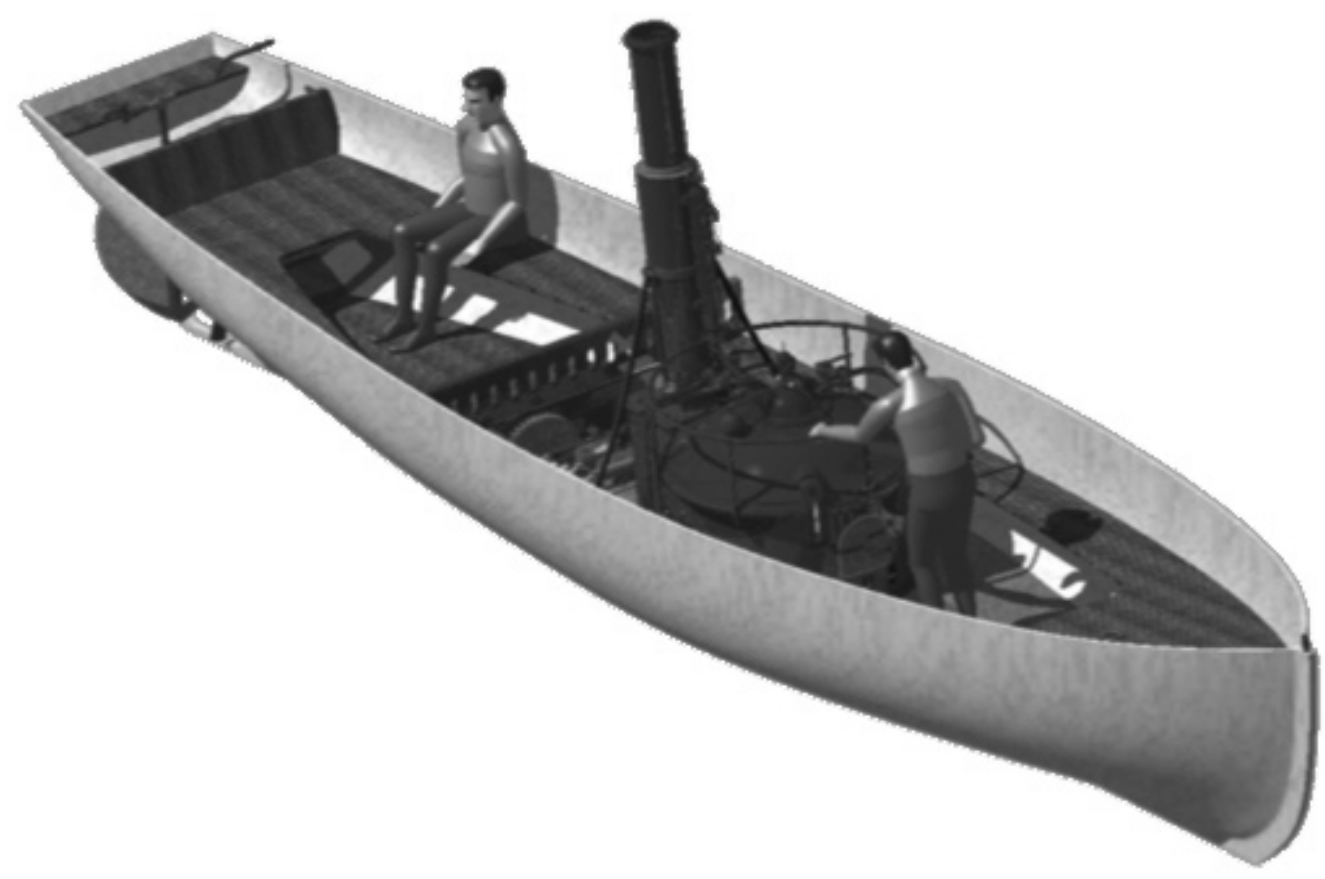

fig. 5 - Canot à vapeur, maquette numérique sous Catia V5.

le logiciel de Dassault Systèmes Catia V5 (fig. 5). De plus, des connaissances externes rassemblées par les historiens ont permis de comprendre et de modéliser les détails invisibles du plan (intérieurs des injecteurs, pompes...) et de connaître les matériaux utilisés. Ces précisions sont obtenues à travers les rapports d'essais de ce prototype ou les correspondances avec le ministère de la Marine, celles-ci donnant par exemple la puissance approximative de la machine, mais aussi les commandes réalisées (marque de l'injecteur, matériaux) qui, croisées avec d'autres documents anciens permettent d'obtenir une définition précise des sous-ensembles cachés du plan.

Enfin, parmi les nombreux projets, nous menons actuellement une étude sur une expérience ayant débuté il y a 20 ans suite à une rencontre fortuite avec un objet technique industriel : une machine artisanale du XXe siècle (1914-1966). II s'agit d'une des dernières laveries à sel de la presqu'île guérandaise. Celle-ci fait partie du parcours muséographique du futur Musée de France des marais salants de Batz-surMer (fig. 6).

La démarche de conservation ef d'investigation entamée pour comprendre cet objet a alors permis de répondre à de nombreuses questions : comment sauver cet objet mobilier fait de métal et de bois et fortement détérioré par le sel ? Comment fonctionnait-il ? Quelle était sa capacité de traitement ? Pourquoi laver le sel de Bretagne ? Comment I'utilisait-on ? Pourquoi le lavage s'est-il arrêté et pourquoi commercialise-t-

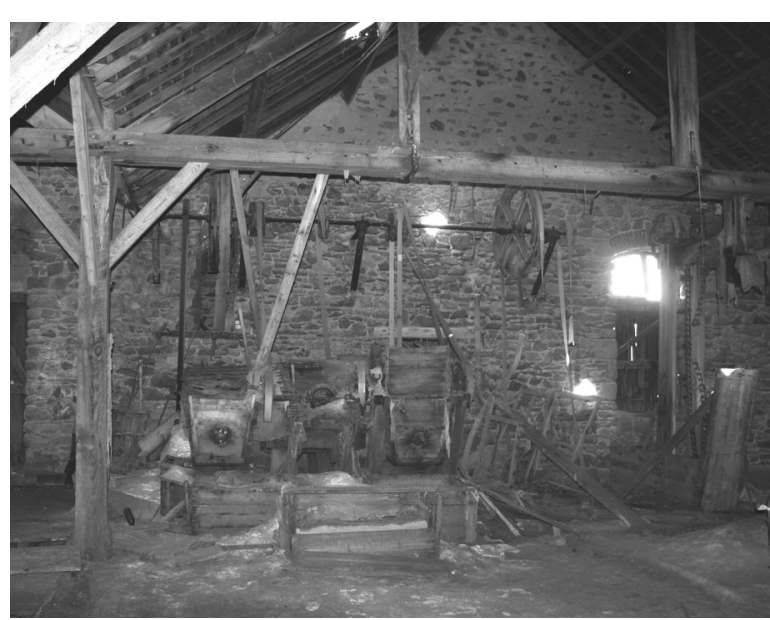

fig. 6-Machine à laver le sel, vestiges archéologiques.

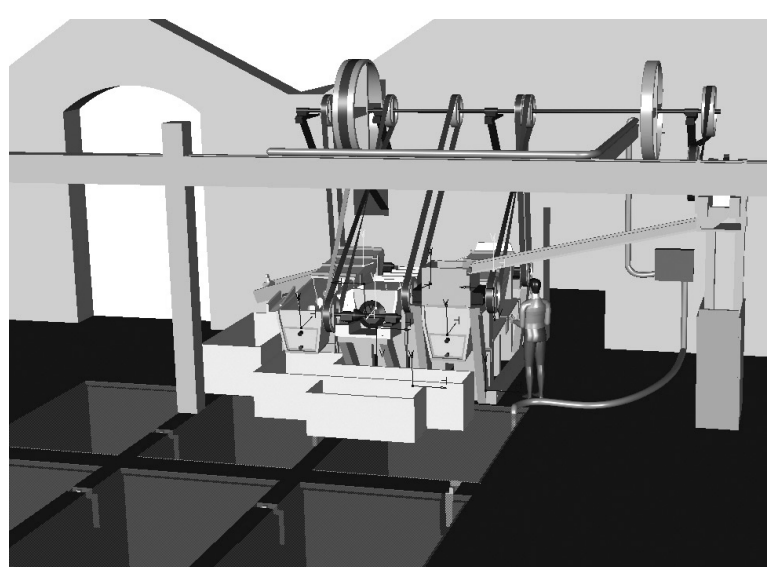

fig. 7 - Machine à laver le sel, maquette numérique. on aujourd'hui du sel gris ? Mangerait-on de nos jours 
du sel impropre ? La prise en compte d'un système technique complet dans ses multiples aspects sociaux, économiques, industriels a alors permis de comprendre l'évolution des valeurs transmises par ce produit de consommation courante.

Après une première phase d'analyse, la capitalisation des connaissances intrinsèques de l'objet a permis de délivrer le savoir-faire nécessaire pour concevoir et utiliser la machine à sel. Ainsi, sa modélisation sous la forme d'une maquette numérique 3D a permis de déduire des informations sur les flux de production mis en œuvre : quantité de sel lavé recueilli, lavé, vendu, exporté (fig. 7) ${ }^{14}$.

Mais cette machine faite de bois et de métal est dans un état de dégradation tel qu'il fut impossible de la sauvegarder ; un démantèlement était donc inévitable. Malgré tout, la problématique soulevée par cette découverte pour tenter de répondre à la question «pourquoi laver du sel ? » a convaincu les conservateurs d'inscrire cette nouvelle étape dans l'histoire dusel. Cerebondissementtechnico-historique va être intégré au parcours muséographique du futur musée. En effet, dans le cadre de l'agrandissement du Musée des marais salants de Batz-sur-Mer, il a été décidé de reconstruire l'objet à échelle 1. Les travaux sont en cours et suscitent de nombreuses questions de la part des artisans en charge de la reconstruction : la machine doit-elle re-fonctionner réellement ? Où retrouver une vis dans le catalogue actuel ? etc.

Notre problématique de recherche permet, le temps d'un instant, de voyager dans d'autres mondes de la technique en revisitant d'anciennes techniques ou des sites industriels oubliés, peu connus, voire inconnus. Les études d'archéologie industrielle menées ont été réalisées en collaboration avec de multiples acteurs du bassin nantais : aussi bien pédagogiques qu'industriels ou associatifs. Les équipes projets interdisciplinaires ont mis en œuvre des connaissances appartenant au présent de la conception, mais également au savoir-faire passé. En réinjectant cette " nouvelle " culture technique dans nos modes de conception contemporains, l'archéo-conception pourrait devenir un tremplin pour l'innovation industrielle ou une nouvelle forme d'enseignement technologique par l'histoire.

Analysons maintenant quelques pistes de développement futur de cette thématique de recherche.

14 Florent Laroche, « Les outils du virtuel : la mécanisation du lavage du sel à Batz-sur-Mer au XXe siècle ", L'archéologie industrielle en France, $n^{\circ} 51$, 2008, pp.46-51.

\section{Perspective... reconstruire les objets d'autrefois ?}

Si la maquette numérique de référence a été suffisamment détaillée depuis un niveau microscopique à un niveau macroscopique, elle peut permettre de reconstruire l'objet technique ancien avec toutes ses qualités physiques initiales.

En effet, la reconstruction apparaît comme une question importante, notamment pour les partenaires avec qui nous avons mené les études de cas. Face à une machine en ruine mais dont l'étude archéologique industrielle avancée a abouti, la réalisation de la maquette numérique de référence montrant un objet en état de marche et fonctionnant virtuellement a créé le désir d'un retour au réel en envisageant une refabrication à échelle réduite ou réelle, fonctionnelle ou non. Ainsi, la capitalisation des connaissances par l'outil numérique pourrait également permettre de reconstruire l'objet ou de le restaurer en réalisant les pièces manquantes.

Cependant, il convient de veiller à ce que la restauration ou reconstruction soit réellement viable. À titre d'exemple, nous avons mené une étude de cas sur une machine à vapeur de marque Piguet (1898-1972). Or, après étude socio-technique, il est apparu que l'objet avait été mal entretenu durant sa troisième phase de vie. Une remise en service des pièces d'origine sans changer les éléments critiques non visibles sur les vestiges de l'objet aurait donc été dangereuse pour le public du musée.

\section{Perspective... la maquette numérique virtuelle comme médiateur?}

La pratique des collections de machines débute au XVIII siècle en Europe et leur utilisation est peu à peu destinée à la formation technique au cours du XIXe siècle, ainsi au Conservatoire des arts et métiers. D'autres créations suivent, tel que le Science Museum à Londres, fondé dans le sillage de l'exposition du Crystal Palace ${ }^{15}$. Les maquettes matérielles encombrantes, dans ces musées, souvent animées, sont de véritables chefs d'œuvre et d'une haute fidélité technique. Cependant, elles sont actuellement orientées vers des buts uniquement muséographiques, suite à la crise de la collection systématique des machines et des objets techniques au XXe siècle.

De plus, en règle générale, les collections sont présentées statiquement, ce qui se comprend fort bien pour des questions de coût, de compétences techniques, d'entretien et d'usure sur le long terme compte tenu de

15 John Liffen, « Le Patent Office Museum, 1857-1883, précurseur du Science Museum de Londres "I, La Revue du Musée des arts et métiers, 51/52, 2009, à paraître. 
leur utilisation. À ces fins, certains musées ou écomusées ont développé des efforts importants de maintien en service de machines et du savoir-faire de leur usage par d'anciens ouvriers capables de faire des démonstrations. De tels choix muséographiques sont néanmoins rares et tendent à disparaître avec l'âge des acteurs. De plus, le maintien des ateliers et des outillages d'entretien est également problématique.

Enfin, les maquettes réelles, statiques ou animées des musées paraissent aujourd'hui démodées pour les jeunes générations, avides d'imageries numériques, qui préféreraient utiliser ces vieilles machines dans Second life ${ }^{16}$.

Les maquettes numériques de référence peuvent alors venir compléter les inventaires et les collections de machinesanciennesdupatrimoinetechniqueetindustriel. En effet, à long terme, grâce à une implémentation de type contributive, un thésaurus de machines constitué d'une base de données conséquente serait appelé à devenir une collection numérique destinée à la conservation de la connaissance des processus industriels, renouant ainsi les liens avec les objectifs originaux des collections de machines.

Les applications didactiques paraissent aussi nombreuses et des plus intéressantes pour la formation technique et scientifique. L'usage de la CAO comme produit pédagogique est devenu une méthode d'apprentissage courante pour la formation des futurs techniciens, ingénieurs. Notre proposition offre une extension de ces pratiques en intégrant la compréhension du passé, pour des démarches de culture métier ou de culture produit, au sein des écoles comme des entreprises. En effet, les études de cas que nous avons menées dans un cadre universitaire ont montré que les étudiants et les enseignants-chercheurs ayant réalisé des projets de maquettes numériques de patrimoine dans des formations de conception mécanique ou d'apprentissage de la CAO, en ont perçu tout l'intérêt pratique. L'utilisation de moyens virtuels sur des vieilles machines pourrait permettre d'anticiper et donc prévenir les pannes, rendre réelle la disponibilité de la machine et accélérer le processus de formation, surtout si ce sont les étudiants eux-mêmes qui déroulent le procesus de patrimonialisation : la confrontation de plusieurs disciplines, de plusieurs langages est alors riche en enseignements.

Perspective... la réalité virtuelle entre dans les musées Une application du dossier d'oeuvre patrimoniale technique peut également être envisagée à des fins de

16 Second life : monde virtuel en 3D, imaginé, créé et possédé par ses joueurs sur Internet ; www.secondlife.com valorisation du patrimoine technique et industriel pour le grand public. Elle interviendra soit comme élément de complément et de mise en contexte d'objets de référence, soit comme projet de restitution. Au vu de la croissance exponentielle des technologies de réalité virtuelle, une approche du patrimoine par les moyens modernes est désormais envisageable : visualisation en trois dimensions, retours haptiques pour toucher les objets virtuels, immersion dans des usines virtuelles où le visiteur prend la place des ouvriers et fait fonctionner l'atelier. Une nouvelle déclinaison de la maquette numérique de référence pourrait donc se concrétiser par la réalisation de parcours muséographiques grand public dans le cadre des missions ministérielles nationales de diffusion de la culture scientifique et technique.

Outre l'intérêt que de telles expositions permanentes procureraient aux experts et aux étudiants en fournissant un modèle rigoureux expliquant les solutions techniques dans leur contexte, les premières pistes évoquées lors de discussions animées avec des conservateurs de musées intégrés à nos projets pourraient être de :

- s'arrêter à une maquette numérique de référence se déclinant en fonction des publics ou des utilisateurs,

- restituer l'environnement des objets avec leurs atmosphères et leurs ambiances (sons, lumières, odeurs), jover sur les sens pour toucherl'émotionnel-une immersion qui susciterait connaissance, étonnement, plaisir,

- découvrir l'objet en entrant à l'intérieur, en se l'appropriant, en se perdant dedans,

- et pourquoi ne pas envisager un objet de création artistique contemporaine ou un spectacle autour de l'objet.

Les potentialités d'expansions muséographiques du dossier d'oeuvre patrimoniale technique sont nombreuses. Daniel Thoulouze, directeur du Musée des arts et métiers à Paris, estimait ainsi que «le musée ne doit pas être qu'un rétroviseur vers le passé mais un tremplin pour l'avenir et l'innovation $)^{17}$.

\section{Perspective... la culture technique, un tremplin pour l'innovation?}

Dans les pratiques industrielles et les démarches d'innovation, les attitudes courantes consistent souvent à sous-estimer, voire à ignorer les acquis du passé. L'image d'une entreprise vieillissante auprès de ses clients est particulièrement redoutée par le service commercial. Les relations entre les préoccupations du marché, les perspectives d'innovation avec le patrimoine industriel et la culture d'entreprise ne sont pas toujours évidentes à concilier. Tout se passe

17 Daniel Thoulouze, « Le patrimoine scientifique et technique contemporain "), (journée d'étude, Musée des arts et métiers, Paris, 2005), publication prévue sous forme électronique pour 2010. 
comme s'il y existait une coupure irrémédiable entre passé et présent, le seul lien établi avec le présent s'effectuant avec le futur immédiat.

La démarche que nous proposons pourrait être une alternative au problème de conservation et de stockage des machines technologiques et industrielles. En effet, comme expliqué précédemment, ce problème a été rencontré non seulement par le musée des arts et métiers mais également par des musées similaires en Allemagne, en Grande-Bretagne ou aux ÉtatsUnis, environ à la même époque. Ce décrochage, essentiellement lié au développement exponentiel des techniques industrielles $d u X X$ siècle, rend particulièrement difficile l'accumulation de ces objets. Un thésaurus virtuel complétant les collections d'inventaires de machines pourrait s'avérer utile, non seulement pour les historiens des techniques mais également pour les industriels, afin d'identifier le contexte et les moteurs de l'innovation. L'approche patrimoniale peut alors compléter les actions classiques de capitalisation des connaissances et d'intelligence économique dans une branche professionnelle donnée. Cette consistance culturelle et historique améliorerait ainsi leurs compétitivités. II s'agit dès lors d'une veille technologique d'un nouveau type prenant en compte la filière technique sur la longue durée et permettant de comprendre certaines inflexions, évolutions ou tendances de l'innovation.

Que les connaissances soient explicites ou implicites, orientées produit ou processus, l'innovation actuelle quasi journalière des entreprises contemporaines n'en reste pas moins incrémentale. En effet, l'innovation de rupture est beaucoup plus difficile à mettre en œuvre et nécessite de nouveaux outils, de nouvelles méthodes et requiert avant tout des connaissances et des compétences diverses. Envisager le passé pour prévenir et créer l'avenir pourrait être une utilisation ultime de la capitalisation de ce patrimoine : innover à partir des connaissances patrimoniales ? N'y-a-t-il pas ici une contradiction épistémologique qui ne serait en réalité qu'une nouvelle vision de penser le futur?

\section{Conclusion}

L'idée d'une utilisation des outils numériques pour l'histoire des techniques a été initiée en 1999 par Michel Cotte alors en charge de la formation humaine de futurs ingénieurs. Plus de dix ans après, le bilan est plus que positif. L'hypothèse a été validée en théorie et en pratique. Même si la démarche demeure encore artisanale, elle tient avant tout dans la rigueur scientifique de l'approche que notre équipe formalise de jour en jour.
Diffuser ces travaux et proposer cette méthode de travail est désormais devenu notre axe de développement principal. Mieux comprendre notre monde, anticiper les évolutions socio-économicotechniques sont nos objectifs, mais au lieu de travailler sur des objets présents, notre proposition consiste à prendre comme point de départ des objets techniques appartenant au passé. C'est dans une vision du futur et une logique d'anticipation, à partir des techniques du passé, que nos projets de recherche sont menés, au sein d'une équipe multi-disciplinaire. Ne pourraiton pas imaginer dans l'avenir des écoles d'ingénieurshistoriens?

Ces interférences entre l'histoire, les techniques et le futur qui sous-tendent nos recherches actuelles, s'inscrivent dans l'histoire de la technologie. Nos travaux perpétuent ainsi l'oeuvre des nombreux vulgarisateurs scientifiques des siècles passés, tel Louis Figuier qui, dans Les merveilles de la science, rêvait d'une technologie générale déployée sous l'égide d'une histoire héroïque des inventions :

« Nous avons étudié le monde ancien, la littérature, l'histoire et la philosophie de la Grèce ou de Rome. Nous sommes parfaitement initiés à l'histoire d'Alexandre et de César, aux faits et gestes de Caton l'ancien et de Denys le tyran, et nous pouvons dire le nombre de galères qui figuraient à la bataille de Salamine. [...] Mais la science est entrée, de nos jours, dans toutes les habitudes de la vie, comme dans les procédés de l'industrie et des arts : nous voyageons par la vapeur ; tous les mécanismes de nos usines sont mus par la vapeur; nous correspondons au moyen d'un courant électrique ; nous commandons notre portrait à la chimie ; nous nous faisons éclairer par un gaz emprunté à la chimie ; c'est la chimie qui conserve nos légumes pour la saison de l'hiver; nous demandons à l'électricité de remplacer nos sonnettes... [...] On ne peut trouver une matière plus intéressante que I'histoire et la description des grandes inventions scientifiques dans lesquelles éclate toute la grandeur du génie humain. [...] Lorsque l'utilité des travaux de ce genre sera mieux appréciée qu'elle ne l'est encore, d'autres écrivains compléteront cette tâche en embrassant l'ensemble tout entier des conquêtes scientifiques de notre époque, et ainsi seront sauvés de l'oubli des monuments précieux qui seront un jour les vrais titres de gloire de l'humanité $\|^{18}$.

18 Louis Figuier, Les merveilles de la science, Préface, tome 1 , Paris, Librairie Furne, 1896, p.2. 